\title{
Incidence of vasculopathy in children with hypothalamic/chiasmatic gliomas treated with brachytherapy
}

\author{
U. Tacke $\cdot$ D. Karger $\cdot$ J. Spreer $\cdot$ A. Berlis • \\ G. Nikkhah $\cdot$ R. Korinthenberg
}

\begin{abstract}
Introduction External brain irradiation in children can cause cognitive decline, endocrine dysfunctions and second malignancies. A rare complication is cerebral vasculopathy, which occurs most often in patients with neurofibromatosis type 1. Interstitial radiotherapy using transient Iodine-125 implants is a radiotherapy option, called brachytherapy, offering excellent survival rates, but little is known on treatment-related morbidity, especially long time vascular changes.

Patients and methods Thirteen children with low-grade hypothalamic gliomas, four of them with neurofibromatosis type 1, were diagnosed and treated at the University Hospital Freiburg, Germany. They belong to a larger group of 44 children with suprasellar low-grade gliomas, treated with transient Iodine- 125 seeds and include those who attended all routine follow-up examinations in Freiburg. After written informed consent from the parents or caregivers all patients underwent magnetic resonance imaging with angiographic techniques in 2001, 3 to 13 years after treatment.
\end{abstract}

U. Tacke $(\bowtie) \cdot$ D. Karger $\cdot$ R. Korinthenberg

Division of Neuropediatrics and Muscular Disorders,

Department of Pediatrics and Adolescent Medicine,

University Hospital Freiburg,

Mathildenstr 1,

79106 Freiburg, Germany

e-mail: uta.tacke@uniklinik-freiburg.de

J. Spreer $\cdot$ A. Berlis

Department of Neuroradiology, University Hospital,

Freiburg, Germany

G. Nikkhah

Department of Stereotactic and Functional Neurosurgery,

Neurocenter, University Hospital,

Freiburg, Germany
Results and discussion Six out of 13 revealed cerebral vasculopathies, only one of them revealed symptoms of intermittent cerebral ischemia. Neurofibromatosis type 1 was present in one affected patient. The aetiology of the cerebral vascular changes is not fully understood so far. Tumour encasement, surgical damage and brachytherapy may contribute as a single risk factor or in combination. To get more information, we recommend MRA for artery vasculopathy at follow-up in all patients with suprasellar brain tumours irrespectively to their former treatment or presence of cerebrovascular symptoms.

\section{Introduction}

Treatment strategies for low-grade tumours in childhood are controversial, since the tumour's behaviour is unpredictable and the patient's prognosis is always unclear. Intervention is imperative for symptomatic tumours, considering the child's age, the tumour's volume and site. Since the 1990s of the last century, treatment follows standardized protocols: First-line treatment is surgery, but often complete tumour resection is not feasible and additional therapeutic approaches such as radiotherapy and chemotherapy are recommended, depending on the child's age and neurofibromatosis type 1 condition $[1,2]$.

External fractionated radiotherapy prevents tumour progression, but is known to cause intellectual impairment, secondary malignancies and endocrine dysfunction, particularly in the younger child [3]. Cerebral vasculopathy, affecting large and 
small cerebral vessels, has been reported as a rare complication after external radiotherapy in adults, as in children, radiated for suprasellar tumours like optic pathway gliomas $[4$, 5]. These complications became evident by stroke or transient ischemic attacks months to years after treatment. Children with neurofibromatosis type 1 (NF1) have an increased risk for these cerebrovascular complications [6-8].

In recent decades newer radiotherapy techniques have been applied for unresectable or progressive childhood brain tumours, one of it is brachytherapy (BT), using transient radioactive Iodine-125 seed implantations. Compared with external radiotherapy, BT has been reported to achieve equivalent tumour control with fewer side effects [9-11], but risks for later vasculopathy are unknown so far.

We initiated a retrospective study on children with symptomatic hypothalamic/chiasmatic low-grade gliomas, diagnosed before the era of standardized treatment protocols. All had transient Iodine-125 implants and were evaluated via magnetic resonance angiography (MRA) years after treatment. We focussed on the incidence and symptoms of cerebral vasculopathy in these patients and evaluated further risk factors like neurofibromatosis type 1 .

\section{Patients and methods}

After informed consent of the parents or caregivers, we analysed data of all 13 paediatric patients with hypothalamic or chiasmatic low-grade gliomas, who came for their regular follow-up between January 1 and December 31 in 2001. Four children had neurofibromatosis type 1 . All had been diagnosed and treated at the University Hospital Freiburg, Germany, between 1988 and 1999. They represent a subcollective of 44 patients with low-grade gliomas who all had BT and who had been described elsewhere [12]. Exclusion criteria from the whole group were: age above 18 years (eight patients), incomplete or no follow-up data in our institution (3 and 16 patients, respectively), BT less than 6 months before (three patients) and death of one patient. The final collective can be viewed as a random sample of all children with low-grade suprasellar gliomas, treated at our institution with BT.

Patient median age at diagnosis was 54 months (range 11 to 129 months). Tumour symptoms were hydrocephalus, visual impairment and endocrine dysfunctions such as pubertas praecox and growth retardation. All had cerebral imaging at diagnosis: magnetic resonance imaging (MRI) in nine, computerized tomography in four patients. They underwent stereotactic serial biopsies for histology prior to treatment, which revealed suprasellar low-grade astrocytoma $\mathrm{WHO} \mathrm{I}^{\circ}$ in 12 patients (pilocytic astrocytoma) and astrocytoma $\mathrm{WHO} \mathrm{II}^{\circ}$ in one girl.

Treatment options were chosen individually based on parameters, such as the clinical symptoms, the child's age and the tumour histology, site and volume. All patients underwent brachytherapy with Iodine-125 implants since complete tumour resection was not feasible. Four children had undergone subtotal tumour resection prior to BT, five patients had two Iodine-125 seed implantations and one patient had adjuvant chemotherapy.

The tumours had a spherical shape of $5 \mathrm{~cm}$ in diameter or less. Low-activity Iodine-125 implants (Amersham, Inc., model 6,702) were placed stereotactically in the centre of the tumour and removed after 25 days. Radiation dose was $60 \mathrm{~Gy}$ at the tumour's margin. Dosimetry was done via STP-4 programme (Stryker-Leibinger, Freiburg, Germany).

Regular follow-up included medical history, complete neuropediatric examination and laboratory tests for blood counts, liver enzymes and endocrine parameters. MRI including T1, T2 and Flair sequences was performed at regular intervals 3, 6, 12 and 24 months after diagnosis or at tumour relapses. Additionally, MRA was performed in all patients at a median interval of 7 years (range 3 to 13 years) after radiotherapy on a 1.5-T scanner (Magnetic Vision, Siemens). A 3D-time-of-flight sequence was acquired with the following parameters: TR $35 \mathrm{~ms}$, TE $7.2 \mathrm{~ms}$, flip angle $20^{\circ}$, field of view $200 \mathrm{~mm}$, matrix $200 \times 512.3$ slabs, slab thickness $48 \mathrm{~mm}$, number of partitions 32, resulting in an effective slice thickness of $1.5 \mathrm{~mm}$. From the axial source images, 3D reconstructions were calculated using maximum intensity projection algorithms. 3D reconstructions and source images were taken into consideration when evaluating.

\section{Results}

In our group of 13 children, vascular pathologies of the circle of Willis or the large brain arteries were found in six patients (see Figs. 1, 2, 3). In two patients, one artery was stenotic. Two patients showed multiple arterial alterations. Aneurysms were found in two patients, one of them had a stenosis and an aneurysm at different vessels. Stenoses were most often seen at the anterior cerebral artery (ACA, four patients); less frequently in two patients, the posterior cerebral artery (PCA), internal carotic artery (ICA) and/or medial cerebral artery (MCA) were involved. One patient died (patient 6). See Table 1 for patients' characteristics and vascular changes.

At BT, median age of all children was 54 months (11129 months). The children with vascular pathologies were 44 months median age (range 11 to 86 months) compared with 73 months (range 20 to 129 months) in the nonaffected group. Age difference between any group was not significant $(p=0.41)$. IR was the only treatment in four of them (patients 2, 3, 5 and 6), two underwent IR after subtotal tumour resection (patients 9 and 11). Tumour encasement was present in two patients (patients 2 and 6). Both had IR as the only treatment, and arterial damage was close to cystic parts of the tumours. Radiation doses did not 


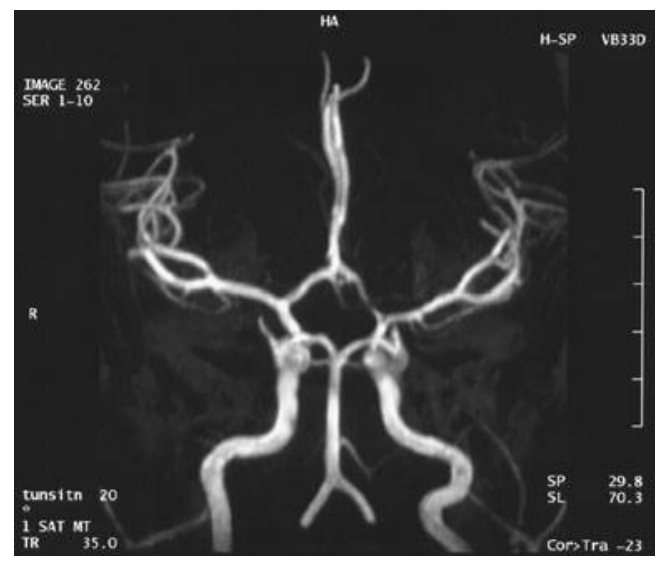

Fig. 1 Asymptomatic stenosis of the left ACA, patient 2

differ between the patients with and without vascular changes. Three patients in both groups had IR twice. Individual dosimetric calculations at the affected vessels revealed maximum radiation doses of 40-50 Gy (patient 3 ), 20-30 Gy (patient 5), 40-50 Gy (patient 9) and 30-40 Gy (patient 11).

Maximum time intervals from interstitial Iodine-125 implants and MRA were 3 to 13 years (median 8 years) in the whole group. Intervals from BT were 6 to 9 years (median 7.8 years) in the affected group compared with 3 to 13 years (median 6 years) in the non-affected group. Differences were non-significant.

Only one patient in the affected group presented with symptoms of vasculopathy such as severe headaches and visual TIAs (patient 11). Four children had neurofibromatosis type 1 , one of them revealed cerebrovascular pathology (patient 2).

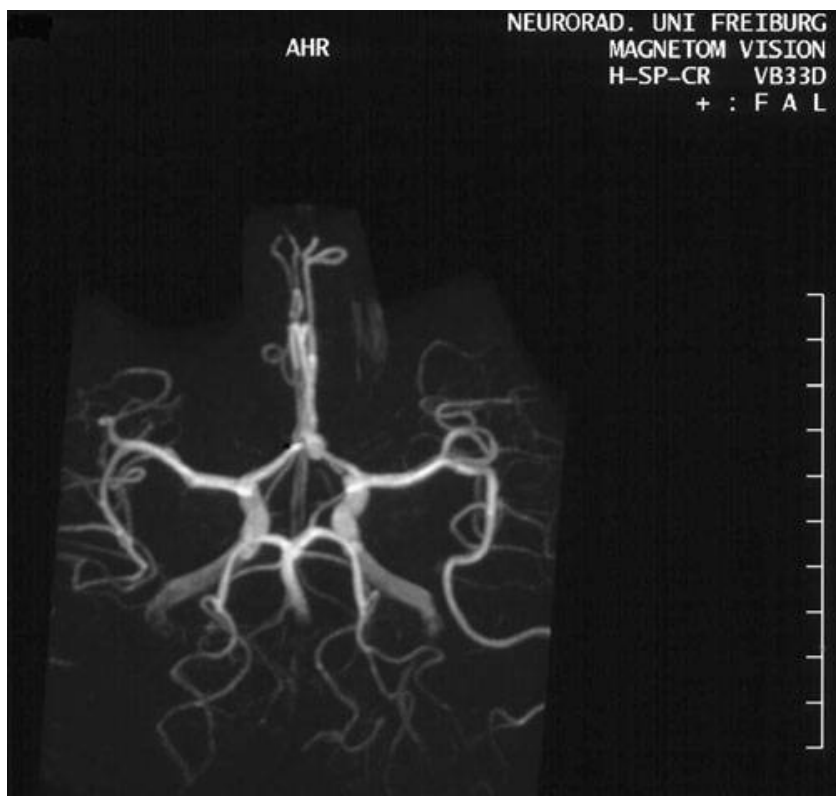

Fig. 2 Asymptomatic aneurysm at the left ACA, patient 5

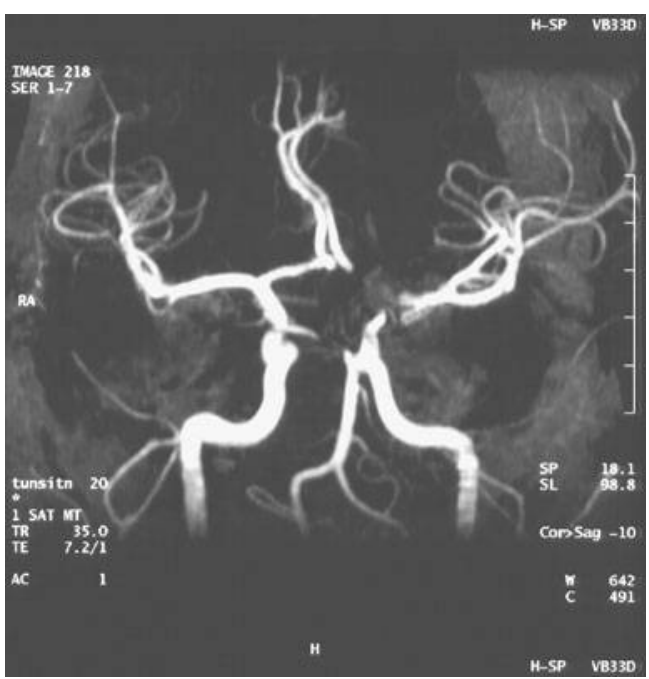

Fig. 3 Multiple stenoses and aneurysm, symptomatic patient 11

\section{Discussion}

We present data on paediatric patients with suprasellar lowgrade gliomas, diagnosed and treated in the 1980s and 1990s of the last century at the University Hospital of Freiburg. All were treated with transient Iodine-125 seed implantations to cumulative doses of 60 Gy (brachytherapy) to avoid or postpone external radiotherapy. These patients represent a subcollective of a larger group from all over Europe, since the University Hospital has a long experience in brachytherapy [11]. Only those patients who attended their regular follow-up in our hospital were included into this study; no other selection criterion was used. They underwent magnetic resonance angiography in 2001 to screen for cerebrovascular changes, a rare complication which has been seen in children following external radiotherapy [4].

All tumours were symptomatic and needed treatment and histology revealed low-grade gliomas. These tumours have an excellent prognosis concerning survival rates, visual and endocrine impairment and tumour progression after total tumour resection. In opto-chiasmatic midline location though, complete resection is not feasible and prognosis is less favourable compared to tumours in cerebellar or hemispherical locations [13].

We reinvestigated data from the 1980s of the last century from paediatric patients that underwent brachytherapy to replace or postpone external brain irradiation. Today, standardized treatment protocols recommend chemotherapy for unresectable tumours in children younger than 8 years or positive for NF1, which offers 10 years of survival rates at about $80 \%$ to $90 \%[14,15]$. But still today, there is a group of patients, who do not respond to chemotherapy or who present with tumour relapses after chemotherapy, so it is of interest to analyse alternatives, like brachytherapy with 


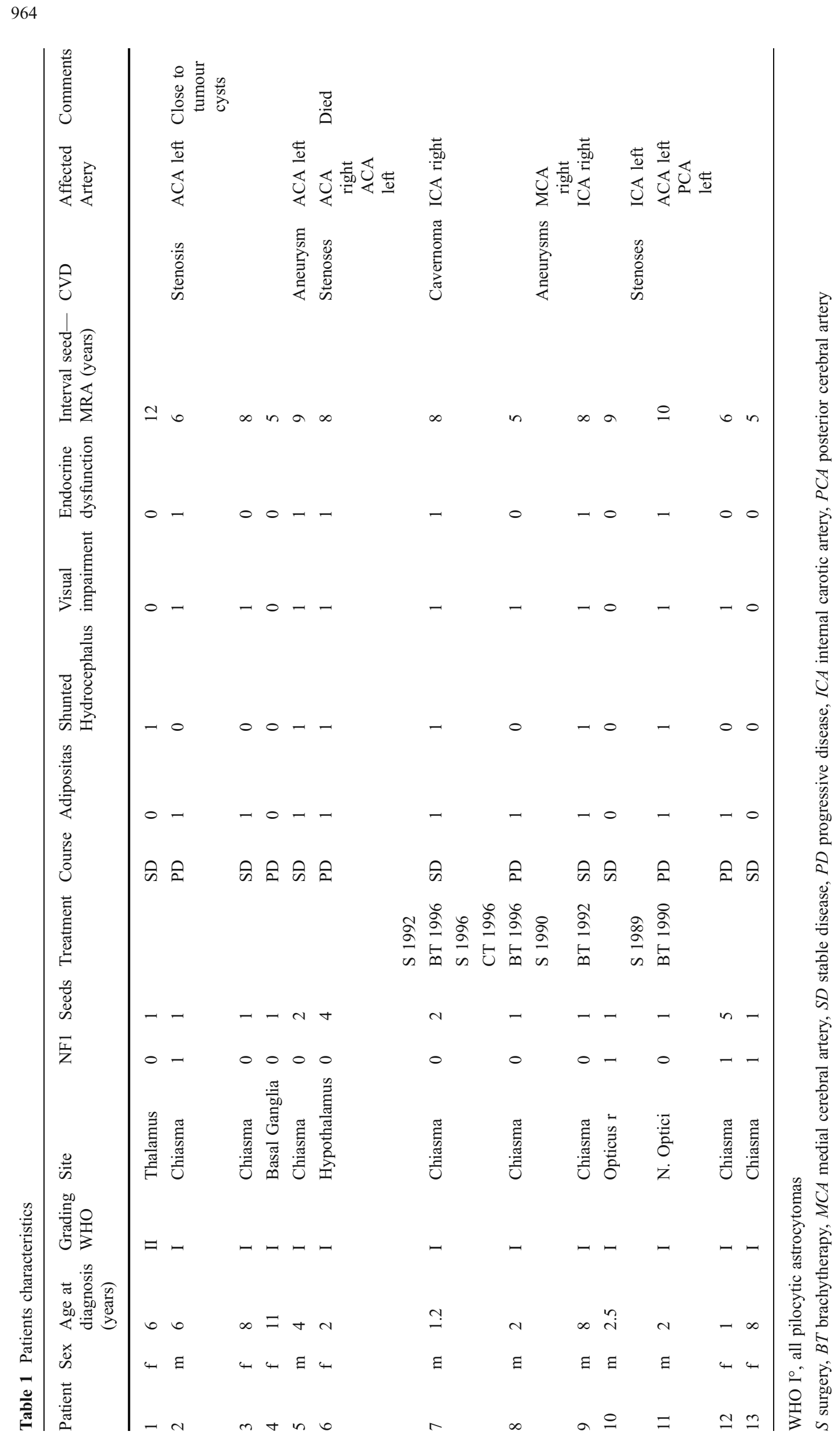


transient Iodine-125 seed implantations in the centre of the tumour and removed after 25 days. This treatment can be a valuable option for selected patients and adults with brain tumours less than $5 \mathrm{~cm}$ in diameter, since it offers equivalent survival rates as surgery and standard radiotherapy [12], and hopefully, reduces radiotherapy-related side effects. These sequelae are mostly seen in long-term paediatric brain tumour survivors after radiotherapy at doses above $30 \mathrm{~Gy}$, again at higher risk for NF1 patients $[16,17]$.

All patients underwent MR imaging with angiographic techniques to evaluate vascular pathologies like stenoses and/or aneurysms of the large cerebral arteries. In six out of 13 patients, vascular changes were diagnosed at single or multiple cerebral arteries. The incidence of vasculopathy in our sample is even higher than those reported after external radiotherapy [4, 5]. However, comparisons there are not possible, since diagnostic methods and patient recruitment differed between all studies. They diagnosed cerebrovascular disease based on standard cerebral computerized tomography or MRI without angiographic techniques, whereas we performed MRA, which is much more sensitive for vascular alterations [18]. So we assume that minor vascular changes may have been overlooked by other investigators. To avoid overestimation of vascular stenoses, a well-known risk of this method, we re-evaluated our affected patients at the following examinations and saw identical results.

Differences in patient recruitment may explain the higher prevalence of vascular changes in our study. We screened all patients for cerebral vasculopathy irrespective of their symptoms, whereas others investigated only those who were symptomatic for strokes or transitory ischemic attacks. Since only one of our patients had clinical symptoms like transient attacks of cerebral ischemia, we would have underestimated the rate of vasculopathy to $1 / 13$ instead of 6/13.

We considered additional risk factors for occlusive arterial vasculopathy in suprasellar brain tumours such as neurofibromatosis type 1, tumour encasement and surgical lesions. Neurofibromatosis type 1 is a major risk factor for radiation-related vascular complications [19] that was present in one affected patient. Tumour encasement may be responsible in two children, since MRI revealed cysts close to the affected vessels. But cysts are frequent complications of brachytherapy, so we could not separate tumour from treatment-related cysts.

Four of the affected patients had BT as the only treatment, two following surgery, so surgical damage cannot be excluded completely. But since both of them had vascular alterations at different vessels, close and distant from the tumour's site, surgery can contribute but not fully explain CVD at different vessels. One patient had an ischemic thalamic stroke during the operation, but again, this might not explain the stenoses of ICA, ACA and PCA.

Cerebrovascular sequelae are seen in long-term survivors of childhood brain tumour, and radiotherapy has a 40 times risk of developing a stroke compared to their sibling controls [20]. Arteriostenotic complications after BT have not been on report yet and early adverse effects of Iodine125 seeds are rare, late reactions can happen and no threshold of radiation doses is known. Up to now, these vascular changes seem to depend on multiple causes; one may be interstitial radiotherapy with Iodine-125 seed implants. This seems surprising, since radioactive seeds are located inside the tumour with a sharp decrease at the margins sparing the surrounding brain tissue [21]. But in opto-chiasmatic location, the circle of Willis is close and the vessels' tolerance to irradiation is uncertain.

So we have to conclude that BT can be one additional risk factor for cerebral arterial changes. Even major cerebral vascular stenosis can be asymptomatic in children. In our patient group, NF1 did not increase the risk for cerebral vasculopathy.

So tumour management strategies must scrutinize treatment indications since suprasellar tumours often exhibit benign courses and many do not require any treatment. Therapeutic interventions must be the target on minimizing treatment-related complications. For those benign brain tumours which cause symptoms and call for intervention surgery, IR and chemotherapy should be considered alone or in combination and carefully applied in order to achieve tumour control and preservation of visual function [22]. To obtain representative data, we recommend a prospective imaging study with MRI and MRA for all paediatric patients with brain tumours irrespective of their treatment.

\section{References}

1. Gnekow AK (1995) Recommendations of the brain tumor subcommittee for the reporting of trials. SIOP brain tumor subcommittee. International Society of Pediatric Oncology. Med Pediatr Oncol 24:104-108

2. Gnekow AK, Kortmann RD, Pietsch T, Emser A (2004) Low grade chiasmatic-hypothalamic glioma-carboplatin and vincristin chemotherapy effectively defers radiotherapy within a comprehensive treatment strategy-report from the multicenter treatment study for children and adolescents with a low grade glioma-HIT-LGG 1996 - of the Society of Pediatric Oncology and Hematology (GPOH). Klin Pädiatr 216:331342

3. Erkal HS, Serin M, Cakmak A (1997) Management of optic pathway and chiasmatic-hypothalamic gliomas in children with radiation therapy. Radiother Oncol 45:11-15

4. Grill J, Couanet D, Cappelli C, Habrand JL, Rodriguez D, SainteRose C, Kalifa C (1999) Radiation-induced cerebral vasculopathy in children with neurofibromatosis and optic pathway glioma. Ann Neurol 45:393-396 
5. Mitchell WG, Fishman LS, Miller JH, Nelson M, Zeltzer PM, Soni D, Siegel SM (1991) Stroke as a late sequela of cranial irradiation for childhood brain tumors. J Child Neurol 6:128-133

6. Rudoltz MS, Regine WF, Langston JW, Sanford RA, Kovnar EH, Kun LE (1998) Multiple causes of cerebrovascular events in children with tumors of the parasellar region. J Neurooncol 37:251-261

7. Bowers DC, Mulne AF, Reisch JS, Elterman RD, Munoz L, Booth T, Shapiro K, Doxey DL (2002) Nonperioperative strokes in children with central nervous system tumors. Cancer 94:10941101

8. Omura M, Aida N, Sekido K, Kakehi M, Matsubara S (1997) Large intracranial vessel occlusive vasculopathy after radiation therapy in children: clinical features and usefulness of magnetic resonance imaging. Int J Radiat Oncol Biol Phys 38:241-249

9. Mohadjer M, Etou A, Milios E, Baden R, Mundinger F (1991) Chiasmatic optic glioma. Neurochir Stuttg 34:90-93

10. Kreth FW, Thon N, Siefert A, Tonn JC (2010) The place of interstitial brachytherapy and radiosurgery for low-grade gliomas. Adv Tech Stand Neurosurg 35:183-212

11. Ostertag CB, Kreth FW (1992) Iodine-125 interstitial irradiation for cerebral gliomas. Acta Neurochir Wien 119:53-61

12. Korinthenberg R, Neuburger D, Trippel M, Ostertag C, Nikkhah G (2010) Long-term results of brachytherapy with temporary iodine-125 seeds in children with low-grade gliomas. Int J Radiat Oncol Biol Phys 79(4):1113-1118

13. Fernandez C, Figarella-Branger D, Girard N, Bouvier-Labit C, Gouvernet J, Paz PA, Lena G (2003) Pilocytic astrocytomas in children: prognostic factors - a retrospective study of 80 cases. Neurosurgery 53:544-553
14. Fisher PG, Tihan T, Goldthwaite PT, Wharam MD, Carson BS, Weingart JD, Repka MX, Cohen KJ, Burger PC (2008) Outcome analysis of childhood low-grade astrocytomas. Pediatr Blood Cancer 51:245-250

15. Gnekow AK, Kaatsch P, Kortmann R, Wiestler OD (2000) HITLGG: effectiveness of carboplatin-vincristine in progressive lowgrade gliomas of childhood - an interim report. Klin Pädiatr 212:177-184

16. Merchant TE, Pollack IF, Loeffler JS (2010) Brain tumors across the age spectrum: biology, therapy, and late effects. Semin Radiat Oncol 20:58-66

17. Morris B, Partap S, Yeom K, Gibbs IC, Fisher PG, King AA (2009) Cerebrovascular disease in childhood cancer survivors: a children's oncology group report. Neurology 73:1906-1913

18. Husson B, Rodesch G, Lasjaunias P, Tardieu M, Sebire G (2002) Magnetic resonance angiography in childhood arterial brain infarcts: a comparative study with contrast angiography. Stroke 33:1280-1285

19. Wentworth S, Pinn M, Bourland JD, Deguzman AF, Ekstrand K, Ellis TL, Glazier SS, McMullen KP, Munley M, Stieber VW, Tatter SB, Shaw EG (2009) Clinical experience with radiation therapy in the management of neurofibromatosis-associated central nervous system tumors. Int J Radiat Oncol Biol Phys 73:208-213

20. Anderson NE (2003) Late complications in childhood central nervous system tumour survivors. Curr Opin Neurol 16:677-683

21. Warnke PC, Kopitzki K, Ostertag CB (2003) Interstitial stereotactic radiosurgery. Acta Neurochir Suppl 88:45-50

22. Burzynski SR (2006) Treatments for astrocytic tumors in children: current and emerging strategies. Paediatr Drugs 8:167-178 